\title{
Uma Experiência Americana de P\&D em Polímeros
}

Os EUA é um dos países que mais investe em desenvolvimento tecnológico de polímeros. Grande parte das pesquisas realizadas nesta área envolve o trabalho conjunto de Universidades, Institutos de Pesquisa e setores de P\&D das empresas. $O$ "Center for Polymer Research" da Universidade do Texas em Austin, é um dos mais importantes centros de desenvolvimento de pesquisas em polímeros nos EUA. Seus temas de pesquisa sempre se encontram na fronteira do conhecimento em várias áreas de polímeros. Além dos enfoques científicos abordados nas pesquisas os temas escolhidos são quase sempre vinculados a interesses tecnológicos. O Professor D. R. Paul é diretor do Center for Polymer Research desde 1981 e tem contribuído sobremaneira para seu sucesso. O Prof. Paul é mundialmente reconhecido como importante autoridade científica e tecnológica nas áreas de pesquisa em blendas poliméricas, membranas poliméricas e propriedades de barreira em polímeros. Atualmente é o editor da revista Industrial Research on Chemical Engineering e tornou-se mundialmente conhecido por ter sido editor do livro Polymer Blends (D. R. Paul \& S. Newman,1978). Além de ser autor de várias centenas de artigos científicos, atua como consultor tecnológico para várias empresas do setor de polímeros. Polímeros: Ciência \& Tecnologia entrevista nesta edição o Prof. D. R. Paul para falar um pouco do Center for Polymer Research, assim como, aproveita esta oportunidade para conhecer algumas de suas opiniões a respeito de importantes aspectos de $P \& D$ na área de polímeros.

Como surgiu o Center for Polymer Research da Universidade do Texas em Austin?

O Departamento de Engenharia Química da Universidade do Texas em Austin decidiu iniciar um programa na área de polímeros quando se apercebeu que esta área representava um setor de grande importância científica e tecnológica e não havia ninguém ensinando ou se dedicando à pesquisa nesta área dentro da universidade.

Fui contratado em 1967 para dar início àquele desafio e, nos anos seguintes, a pesquisa sobre polímeros se expandiu através dos vários departamentos da universidade a medida que novos membros do corpo docente foram sendo contratados e outros pesquisadores pertencentes a este corpo passaram a desenvolver pesquisas sobre polímeros. Em 1981 notou-se que havia uma massa crítica de docentes e alunos trabalhando na área de polímeros, porém de forma pouco organizada. Em conseqüência disto meus colegas e eu resolvemos criar o Center for Polymer Research com a finalidade de coordenar e promover a pesquisa e a educação em polímeros de maneira interdisciplinar dentro da Universidade do Texas. Embora o escopo do Centro não inclua o oferecimento de cursos de graduação ou de pós-graduação, docentes associados são responsáveis por disciplinas que proporcionam a educação formal em polímeros, vinculadas a diversos cursos da Universidade do Texas em Austin. As oportunidades de estudos a nível de pós-graduação com ênfase em pesquisa na área de ciência e engenharia de polímeros são oferecidas através de vários programas de pós-graduação. Oportunidades para pós-doutorandos e professores visitantes para realizar estudos avançados em polímeros também estão disponíveis para os interessados. O Centro estimula sempre pesquisas através da cooperação entre docentes e profissionais vinculados a indústrias.

Como são os programas de cooperação entre o Center for Polymer Research e as empresas?

Nossas pesquisas em colaboração com as indústrias ocorrem de várias formas. Em alguns casos, é estabelecido um contrato de pesquisa formal entre a empresa e a Universidade, especificando os termos financeiros, o plano de pesquisa, a propriedade intelectual dos resultados e os termos que asseguram a proteção dos segredos industriais, etc. A empresa neste caso é responsável pelos recursos financeiros, oferecidos na forma de bolsa-de-estudos aos estudantes envolvidos na pesquisa, material 
permanente e material de consumo, e outros custos diretos necessários para a execução do projeto, incluindo um overhead de $51 \%$ relativo ao custo total do projeto.

É muito comum empresas in- direitos de propriedade intelectual.

$$
\text { Como são escolhidos os tópicos }
$$
de pesquisa nos programas de cooperação? Quais são os principais tópicos abordados no momento? sas ou para as agências de fomento com a esperança de conseguir recursos para realizarem seus próprios trabalhos de pesquisa. Em outros casos, uma empresa contata diretamente o membro do corpo docente teressadas em nossos programas de pesquisa em andamento oferecerem recursos, sem a imposição de restrições. Neste caso não existe qualquer contrato estipulando um trabalho específico a ser feito e nem qualquer custo de overhead estabelecido pela Universidade. Muitas vezes as empresas colaboram com os programas de pesquisa doando materiais e serviços para a realização dos trabalhos, sem que haja envolvimento direto de recursos financeiros. Algumas empresas enviam seus funcionários para trabalhar junto com os docentes no Centro por períodos que variam de poucos meses até dois anos, sem qualquer custo para nós.

Existe também uma forma especial de cooperação que é feita através de consórcios de pesquisa. Neste caso um grupo de empresas paga uma taxa anual para financiar pesquisas em uma área geral. Um exemplo deste tipo de cooperação é o Programa de Pesquisa na Área de Separação, em que mantém a maioria das nossas pesquisas sobre membranas. Neste caso existe um contrato geral entre a universidade e cada uma das várias empresas, especificando a área geral de pesquisa e detalhando os

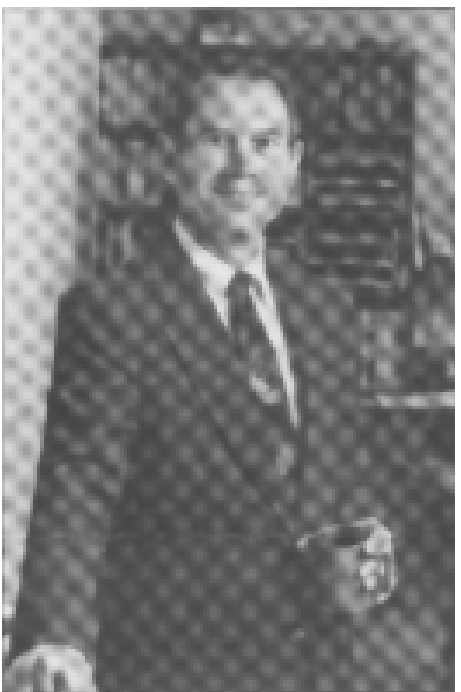

Professor D.R. Paul, fundador e diretor do Center for Polymer Research da Universidade do Texas em Austin

O Professor Paul é um dos mais importantes pesquisadores nas áreas de Ciência e Tecnologia de polímeros. Sua atuação é constatada através da edição de livros, periódicos e publicação de artigos, além de atividades acadêmicas e participação como membro de várias sociedades e entidades relacionadas com polímeros.

Sua atuação como consultor para empresas e órgãos governamentais é mais uma atividade importante dentro de sua vida profissional.

A comunidade americana e internacional tem reconhecido o trabalho do Prof. Paul através de várias homenagens prestadas a ele e vários prêmios, entre os quais citamos Arthur K. Doolittle Award oferecido pela Sociedade Americana de Química (ACS) em 1973, Teaching Achievement Award oferecido pela Universidade do Texas em 1977, Joe J. King Professional Engineering Achievement Award em 1981, Society of Plastics Engineer Award for Outstanding Achievements in Research oferecido pela SPE em 1982, ACS Award in Applied Polymer Science patrocinado pela Phillips Petroleum Co. em 1984, National Academy of Engineering em 1988, Aiche Materials Engineering and Sciences Division Award em 1985, SPE International Award for Outstanding Achievements in Education oferecido pela SPE em 1989, Society of Plastics Engineers International Award oferecido pela SPE em 1993 e Hocott Distinguished Centenarial Engineering Research Award em 1993.

O Prof. Paul já editou 7 livros sobre polímeros, entre eles, Polymer Blends (Dr. Paul \& Newman, Academic Press, 1978, traduzido para o russo e chinês) Multicomponent Polymer Materials (Dr. Paul \& L.H. Sperling, Advances in Chemistry Series $n^{\circ} 211$, ACS, 1986) e Polymeric Gas Separtion Membranes (Dr. Paul \& Y.P. Yampol'skii, CRC Press, 1994). Atualmente ele é editor da revista Industrial \& Engineering Chemistry Research publicada pela Sociedade Americana de Química (ACS), além de participar como membro do Editorial Boards de vários periódicos, tais como Journal of Polymer Science, Journal of Applied Polymer Science, Polymer Contents e Journal of Membrane Science.
Na maioria dos casos, os tópicos de pesquisa refletem o interesse dos docentes associados ao Center for Polymer Research. Cada membro do corpo docente tem uma área de especialidade e de interesse e em muitos casos, eles escrevem espontaneamente propostas para as empre- para desenvolver um projeto de pesquisa de seu interesse. Geralmente a empresa seleciona um membro docente que está trabalhando numa área de pesquisa próxima de seu interesse, entretanto, a sugestão de um tópico por parte da empresa pode ser completamente fora do escopo 
daquela do pesquisador do Centro. Esta é uma importante alternativa para os membros do corpo docente se envolverem com novas idéias a serem pesquisadas.

Os tópicos de pesquisa que estão sendo desenvolvidos pelos associados são os mais diversos; dois deles, de nossa tradição, envolvem difusão em polímeros (com aplicação para materiais de barreira e membranas de separação) e blendas poliméricas. Este último tema inclui assuntos relacionados a miscibilidade, compatibilização e tenacificação através da incorporação de borracha. Existem programas de pesquisa envolvendo o uso de polímeros em aplicações de microeletrônica, particularmente, fotolitografia e fotoquímica. Além destes programas, são realizadas pesquisas nas áreas de biopolímeros, concretos poliméricos, compósitos, etc. $\mathrm{O}$ enfoque mais comum nos diversos temas de pesquisa é a correlação entre estrutura, propriedades e processamento.

Existe alguma fonte principal de recursos para o desenvolvimento das atividades de pesquisa no Center for Polymer Research? Qual é a participação do governo, através de suas fundações, e das empresas no financiamento das pesquisas?

Não temos o controle específico dos dados sobre a origem dos financiamentos, pois os recursos são administrados pelos diversos departamentos vinculados ao Centro. Entretanto, podemos afirmar, com certeza, que o suporte financeiro dos governos estadual e federal é a maior fonte de recursos. $\mathrm{O}$ apoio por parte das empresas equivale de 25 a $35 \%$ do total de recursos para as atividades de pesquisa em polímeros.
Nos programas de cooperação entre o Centro e as empresas, onde existe a geração de patentes, quais são os termos do acordo dos direitos de propriedade intelectual dos resultados?

O direito de patente é um tópico muito importante em todos os contratos de cooperação com as empresas. A posição da Universidade nesta área é ditada por certas leis do estado. Assim, os direitos de propriedade de todas as invenções e descobertas devem ficar com a universidade; porém é resguardado aos patrocinadores da pesquisa o direito de negociar com a Universidade uma licença exclusiva de uso da propriedade intelectual em nível mundial. Assim, podem usar ou comercializar o uso de qualquer invenção ou descoberta feita durante o período da pesquisa contratada. A empresa pode também ter o direito de sub-licenciar a tecnologia gerada durante a pesquisa. Geralmente é dado um prazo de três meses à empresa patrocinadora, após a geração da invenção ou da descoberta, para que notifique a Universidade sobre seu interesse em negociar os termos da licença. Se a empresa e a Universidade não chegarem a um acordo, os direitos de tais invenções e descobertas são concedidos e/ou negociados exclusivamente pela Universidade, sem a participação do patrocinador da pesquisa.

Existem programas de cooperação entre o Center for Polymer Research e outros centros ou institutos de pesquisas nos EUA e no mundo? Como isso ocorre?

O Centro tem realizado cooperação científica e tecnológica com uma variedade de institutos de pesquisa em todo o mundo. A coope- ração ocorre em termos informais sem o envolvimento de contratos específicos para tal.

Considerando sua renomada experiência como pesquisador e consultor na área de blendas poliméricas, o que poderia ser comentado sobre a tendência para o desenvolvimento de blendas poliméricas no futuro? Quais são seus principais desafios científicos e tecnológicos?

O desenvolvimento de blendas poliméricas tornou-se extremamente importante nas últimas duas décadas no setor industrial. Já há algum tempo tem sido considerado que o desenvolvimento de novos polímeros é muito dispendioso e que a alternativa de "fabricação de blendas poliméricas" seja talvez a forma mais adequada de conseguir novos sistemas poliméricos, com novas propriedades, a um custo compatível com o mercado. Entretanto, é importante notar que, a despeito disto, um número considerável de novos polímeros tem surgido frequientemente no mercado. Em minha opinião, o desenvolvimento de blendas poliméricas não deveria ser considerado estritamente como uma alternativa de competição para o desenvolvimento de novos polímeros. Melhor que isso, acho que a fabricação de blendas oferece um meio de alcançar níveis de desempenho que são viáveis através de um único polímero. Por exemplo, produtos obtidos a partir de blendas poliméricas oferecem uma combinação de propriedades (tal como a tenacidade e a resistência química) inviável de ser obtida num único material. Naturalmente plásticos super tenazes requerem uma combinação apropriada de uma fase borrachosa numa matriz rígida. 
O desenvolvimento de blendas poliméricas oferece tantas oportunidades tecnológicas que fica difícil identificar uma única tendência desta área no futuro. Estes materiais são usados como plásticos de engenharia, materiais para embalagem, sistema auto controlado de liberação de medicamentos, etc. Entretanto, eu acho que o foco principal no setor de pesquisa sobre blendas poliméricas será através de um controle mais preciso de interação polímeropolímero, interfaces polímeropolímero, e morfologia de fases da blenda. Nós estamos começando a entender melhor a importância destes tópicos e como eles podem ser mais precisamente controlados.

Poderia nos dar uma idéia de qual o nível de investimento que as indústrias nos EUA estão aplicando em P\&D na área de blendas poliméricas?

Não acredito que alguém possa fazer uma estimativa precisa sobre o nível de investimento em $\mathrm{P} \& \mathrm{D}$ de blendas poliméricas que a indústrias têm feito nos EUA. A principal razão é que as empresas não separam tão distintamente o investimento em $P \& D$, e mesmo se fizessem, esta informação dificilmente seria tornada pública. Entretanto, tenho observado que uma parcela significativa de esforços em pesquisa na área de blendas poliméricas tem sido realizada pela General Electric, Rohm and Haas e Du Pont.

Qual é sua opinião a respeito das tendências no desenvolvimento de membranas poliméricas? Quais seriam os principais desafios do uso de membranas poliméricas nos processos de separação e no uso de barreiras poliméricas como embalagens?
O uso de membranas poliméricas para processos de separação tem aumentado drasticamente nas duas últimas décadas. $\mathrm{O}$ uso de polímeros como membranas para separações de líquidos, como no caso de osmose reversa e ultrafiltração já atingiu um estágio avançado. O principal desenvolvimento nesta área específica, nos últimos tempos, tem sido ampliar a escala de uso de membranas em separações de gases. Os principais nichos nesta área são separação de nitrogênio e de oxigênio do ar, recuperação de hidrogênio a partir de vários processos de corrente de gases, remoção de umidade de corrente de gases, remoção de dióxido de carbono de gás natural, etc. Existe um interesse muito grande no desenvolvimento de novos polímeros para otimizar estes processos e para converter estes novos materiais em membranas de alto fluxo assimétrico ou em membranas compósitos.

Ultimamente, as atividades comerciais nestas áreas tornaram-se muito mais concentradas e novos sistemas de membranas estão sendo introduzidos no mercado. A pesquisa fundamental na área tem abrangido uma ampla faixa de interesses, mas eu acredito que este tipo de pesquisa se tornará muito mais próximo da prática comercial através de um esforço na expansão das oportunidades tecnológicas. Como consequiência das pesquisas realizadas nos últimos 15 anos, eu acho que conseguimos atingir um bom nível de entendimento sobre quais possibilidades e quais limitações existem em aplicações de membranas poliméricas nos processos de separação.

A separação de líquidos através do processo de pervaporação é conhecida a muito tempo; entretanto, a implementação comercial deste conceito tem sido bastante limitada nos dias atuais. Acredito que nos próximos anos existirá um esforço maior em pesquisas nesta área e surgirão processos comerciais deste tipo de separação. Talvez este seja um dos principais desafios para o futuro.

Os polímeros são importantes materiais para o setor de embalagens e suas propriedades de barreira para o oxigênio, água, etc., são críticas nestas aplicações. Existe um grande interesse em materiais poliméricos com melhores propriedades de barreira, pois isto favorece o aumento do "shelf life" para os produtos embalados e proporciona novas oportunidades para embalagem de produtos até então sem alternativas. Avanços graduais vêm sendo continuamente feitos nos polímeros existentes através do ajuste de condições de polimerização e de processamento destes; pequenos avanços nesta área são importantes, pois resultam em significativas oportunidades comerciais. Entretanto, continua a existir uma necessidade de materiais com melhor desempenho em relação às suas propriedades de barreira que não pode ser alcançado pela simples manipulação dos polímeros já existentes.

Para a maioria das aplicações, os materiais para embalagem devem ter baixo custo visto que são descartados e eles necessitam, em muitos casos, serem recicláveis. Nós temos demonstrado em nossos laboratórios que cristais líquidos poliméricos (LCP) quase sempre possuem excelentes propriedades de barreira, mas atualmente seu custo é excessivo e a ausência de transparência o torna inviável em aplicações tipo "commodities". Por outro lado, existe um interesse considerável no uso de cristais líquidos poliméricos em várias situações especiais onde as propriedades de barreira são extre- 
mamente importantes. Nos últimos anos, os processos de produção comercial de poli(naftenato de etileno) (PEN) têm sido desenvolvidos. Estes materiais possuem propriedades

de barreira muito melhores do que o poli(tereftalato de etileno) (PET), entretanto seu custo é significativamente mais elevado. Copolímeros constituídos de PET e PEN deverão ter uma importância comercial muito grande nos próximos anos. Outra área de interesse, mas ainda em estágio prematuro, é a formação de nanocompósitos constituídos de uma matriz polimérica com pequenas plaquetas de elevada razão de aspecto dispersas. Entretanto, os aspectos econômicos destes materiais ainda não foram determinados.

VOLUME I. BLEND FORMULATION
Introduction

General Thermodynamics

Binary Interaction/Mean Field Theory

Hydrogen Bonding Systems

Analog Calorimetry

Crystallization in Blends

Fundamentals of Blends of Rigid Chain (Liquid Crystal) Polymers

Morphology Characterization by Microscopy Techniques

Viscoelastic Characterization

Blend Characterization by Light Scattering Techniques

Neutron Scattering/Reflection

Formulation and Characterization of Thermoset/Thermoplastics Blends

Chemical Reactions in Blends Based on Condensation Polymers

Blends Involving Performed Block Copolymers

Morphology Generation during Melt Mixing Operations

Reactive Compatibilization
D.R. Paul \& C.B. Bucknall I.C. Sanchez

G.D. Merfeld \& D.R. Paul P. Painter e colaboradores C.J.T. Landry

J.P. Runt

M. Ballauff

S.I. Hobbs and V. Watkins

D.S. Kalika

T. Inoue

D.G. Bucknall, W.W.

Graessley \& D.J. Lohse

J.P. Pascault \& R.J.J. Williams

G. Groeninckx \& S. Thomas

A.M. Jamieson

B.D. Favis

B. Majumdar \& D.R. Paul

\section{VOLUME II. BLEND PERFORMANCE}

Quasi-elastic Mechanical Properties

Characterizing Toughness Using Fracture Mechanics

F.J. Guild

Y-W. Mai

C.B. Bucknall

Characterizing Toughness Using Standard Empirical Tests

Deformation Mechanisms in Rubber-Toughned Polymers

C.B. Bucknall

Strengthening Polymer-Polymer Interfaces

Toughening with Core-Shell Impact Modifiers

Toughening Crystalline Thermoplastics

H. Brown

Toughening of Thermosetting Resins and Multiphase Plastic Alloys

C.A. Cruz

R.J. Gaymans

A.F. Yee

Fatigue Crack Propagation in Polymer Blends
Transmission and Reflection of Light in Multiphase Blends

R.A. Pearson

R.A. Katz

J.J. Scobbo

Thermo-mechanical Performance

Chemical Durability of Blends

Barrier Materials by Blending

Reinforced Polymer Blends

Liquid Crystal Polymer Blends

Elastomer Blends

Thermoplastic Elastomers by Blending

Recycling Polymer Blends

A. Berzinis e colaboradores

P.M. Subramanian

J. Karger-Kosics

D.G. Baird

S. Datta

S. Abdou-Sabet \& S. Datta

T.S. Ellis

Obs. Cada assunto corresponde a um capítulo do livro. Deve ser ressaltado que o projeto do livro é tentativo. Durante a publicação do livro poderá ocorrer modificações.

Entrevista realizada pelo Prof. Dr. Elias Hage J r. (UFSCar/DEMa) em colaboração com o Prof. Dr. Luiz Antonio Pessan (UFSCar/DEMa).
Ficamos sabendo que você vai editar um novo livro sobre blendas poliméricas. Poderia falar um pouco a respeito?

O Prof. Clive Bucknall e eu estamos iniciando a edição de um novo livro sobre blendas poliméricas. $\mathrm{O}$ primeiro volume se concentrará em aspectos de formulação, enquanto que o segundo volume será dedicado ao desempenho das blendas; nós estamos esperançosos que este livro venha a ser publicado em 1999. Farão parte do primeiro volume alguns capítulos sobre termodinâmica, caracterização e formação de estruturas em blendas. O segundo volume apresentará alguns capítulos sobre propriedades mecânicas e resistência à fadiga, desempenho específico durante a preparação de blendas, blendas reforçadas, blendas elastoméricas e reciclagem de blendas (vide quadro). 